\title{
Rate-dependent mechanical behaviour of semilunar valves under biaxial deformation: from quasi-static to physiological loading rates
}

\author{
Afshin Anssari-Benam ${ }^{1, *}$, Yuan-Tsan Tseng ${ }^{2}$, Gerhard A. Holzapfel ${ }^{3,4}$ and Andrea Bucchi ${ }^{1}$ \\ ${ }^{1}$ Cardiovascular Engineering Research Laboratory (CERL), School of Mechanical and Design \\ Engineering, University of Portsmouth, Anglesea Road, Portsmouth PO1 3DJ, United \\ Kingdom
}

${ }^{2}$ National Heart and Lung Institute, Heart Science Centre, Imperial College London, Middlesex, United Kingdom

${ }^{3}$ Graz University of Technology, Institute of Biomechanics. Graz, Austria

${ }^{4}$ Norwegian University of Science and Technology (NTNU), Department of Structural Engineering, Trondheim, Norway

* Address for correspondence:

Afshin Anssari-Benam,

Cardiovascular Engineering Research Laboratory (CERL),

School of Mechanical and Design Engineering,

University of Portsmouth,

Anglesea Road,

Portsmouth PO1 3DJ

United Kingdom

Tel: $\quad+44(0) 2392842187$

Fax: +44 (0)23 92842351

E-mail: afshin.anssari-benam@port.ac.uk 


\begin{abstract}
In this study we investigate the rate-dependency of the mechanical behaviour of semilunar heart valves under biaxial deformation, from quasi-static to physiological loading rates. This work extends and complements our previous undertaking, where the rate-dependency in the mechanical behaviour of semilunar valve specimens was documented in sub-physiological rate domains (Acta Biomater. 2019, https://doi.org/10.1016/j.actbio.2019.02.008). For the first time we demonstrate herein that the stress-stretch curves obtained from specimens under physiological rates too are markedly different to those at sufficiently lower rates and at quasistatic conditions. The results importantly underline that the mechanical behaviour of semilunar heart valves is rate dependent, and the physiological mechanical behaviour of the valves may not be correctly obtained via material characterisation tests at arbitrary low deformation rates. Presented results in this work provide an inclusive dataset for material characterisation and modelling of semilunar heart valves across a 10,000 fold deformation rate, both under equibiaxial and 1:3 ratio deformation rates. The important application of these results is to inform the development of appropriate mechanical testing protocols, as well as devising new models, for suitable determination of the rate-dependent constitutive mechanical behaviour of the semilunar valves.
\end{abstract}

Keywords: Semilunar valves; rate-dependency; mechanical behaviour; physiological rate 


\section{Rate-dependent mechanical behaviour of semilunar valves under biaxial deformation: from quasi-static to physiological loading rates}

\section{Introduction}

Traditionally, the mechanical behaviour of valvular tissues has been viewed to be rateinsensitive (see, e.g., Grashow et al. 2006; Stella et al. 2007). In a series of recent contributions, however, the authors have demonstrated for the first time that if tested across sufficiently large span of stretch rates $\dot{\lambda}\left[\mathrm{s}^{-1}\right]$, aortic valve (AV) and pulmonary valve (PV) samples exhibit a marked rate-dependency in their mechanical behaviour under tensile biaxial loading, both with and without preconditioning (Anssari-Benam et al. 2018; 2019). These studies were preceded by earlier contributions from the host group underlining the same rate-dependent behaviour for AV specimens under uniaxial loading (Anssari-Benam et al. 2011a; 2017). Our results have therefore put forward a strong set of evidence in countering the traditional narrative in heart valve biomechanics: the mechanical behaviour of semilunar heart valves is rate-dependent.

In the studies that have thus far investigated and documented the rate-dependency of the mechanical behaviour of semilunar valves under biaxial deformation, rate effects have been characterised and addressed up to stretch rates of $\dot{\lambda}=1 \mathrm{~s}^{-1}$ (Anssari-Benam et al. 2018; 2019), mainly due to technical challenges in experimental setup adaptations. While there appears to be a variation in the literature as regards to the in vivo physiological stretch rate levels endured by functioning heart valves, the common consensus indicates rates notably above the previously employed $\dot{\lambda}=1 \mathrm{~s}^{-1}$ rate. For example, Leeson-Dietrich et al. (1995) report a value of $\dot{\lambda}=2.5 \mathrm{~s}^{-1}$ as the physiological stretch rate experienced by the AV. Based on the work of Thubrikar et al. (1993), Doehring et al. (2004) have calculated in vivo extension rates as high as $75 \mathrm{~mm} / \mathrm{s}$; while Sacks and Yoganathan (2007) report physiological strain rates of approximately $440 \% \mathrm{~s}^{-1}$ and $1240 \% \mathrm{~s}^{-1}$ in the (principal) circumferential and radial loading directions, corresponding to stretch rates of $4.4 \mathrm{~s}^{-1}$ and $12.4 \mathrm{~s}^{-1}$, respectively.

The preliminary evidence on the rate-dependency of the mechanical behaviour of semilunar valves across lower rates than the physiological, and that the physiological rates are reported to be notably higher than the maximum rate employed in earlier studies, further motivate and prompt establishing the rate-dependency in the mechanical behaviour of valvular tissues up to 
the physiological rate. Indications are that the mechanical properties characterised at arbitrary lower rates in vitro will not be an accurate representative of the valve behaviour in vivo. This study therefore extends our earlier work by including and incorporating the physiological rates. We herein investigate and present rate-dependency in the mechanical behaviour of semilunar valves under biaxial loading, from a quasi-static $\dot{\lambda}=0.001 \mathrm{~s}^{-1}$ to the upper end of the reported physiological $\dot{\lambda}=12.4 \mathrm{~s}^{-1}$ rate, encompassing $\dot{\lambda}=1,2.5 \mathrm{~s}^{-1}$ and $6.25 \mathrm{~s}^{-1}$, covering a range of 10,000 fold. A clear rate-dependent trend in the obtained stress-stretch curves of valvular specimens is documented and demonstrated. Important implications of the results of this study for development of appropriate methods of characterisation of the mechanical behaviour, as well as continuum and computational modelling for the determination of the rate-dependent constitutive properties, of heart valves will be conferred and discussed.

\section{Materials and methods}

In this manuscript we report results from 60 porcine AV and PV samples ( $n=60$ for each valve), obtained from animals of 12 to 36 months old, within 2 hours of slaughter from a local abattoir. The AV and PV leaflets were dissected from the hearts and maintained in Dulbecco's Modified Eagle's Medium (DMEM, Sigma, Poole, UK) at room temperature $\left(20^{\circ} \mathrm{C}\right)$. From each leaflet, a $12 \times 12 \mathrm{~mm}$ square sample was excised from the central region. The square samples were then subjected to either equi-biaxial or 1:3 ratio biaxial deformation rate loading protocols up to failure. See Figure 1 for a schematic representation of the setup and samples. Six repeats were carried out for each test at each deformation rate.

After excision, the sample thickness was measured using a non-contact confocal sensor (Micro-Epsilon $\left(\mu \varepsilon^{\circledR}\right)$ confocalDT IFC2451), customised for measurement of the thickness of our samples. The average $( \pm \mathrm{SD})$ measured thickness for AV and PV specimens used in this study reported values of $0.93 \pm 0.31 \mathrm{~mm}$ and $0.71 \pm 0.22 \mathrm{~mm}$, respectively. The samples were then mounted on a TA Instruments ElectroForce ${ }^{\circledR}$ planar biaxial TestBench device using BioRake (CellScale ${ }^{\circledR}$ ) tines as part of a custom-designed sample mounting mechanism. Five ink-markers were placed on the centre of the specimens as fiducials for measuring and calculating the principal stretches $\lambda$, and their centroids were tracked over the period of deformation using in-house video cameras, recording at various frames per second (from 30 to $455 \mathrm{fps}$ ) pertinent to the rate of deformation at each test. The recorded frames were then 
analysed using a custom-developed code in MATLAB $^{\circledR}$ to calculate the values of $\lambda$. The hydration of the samples during each test was maintained by immersion in a PBS bath in situ as part of the experimental setup, kept at room temperature. The details of the processing method as well as the experimental setup can be found in our previous studies (Anssari-Benam et al. 2018; 2019). Prior to the start of each test, a preconditioning protocol consisting of 25 loading-unloading cycles at $0.5 \mathrm{~Hz}$, to amplitudes of $0.5 \mathrm{~N}$ and $0.3 \mathrm{~N}$ was applied on $\mathrm{AV}$ and PV specimens, respectively. Samples were subjected to a tare load of $0.01 \mathrm{~N}$ prior to the start of the deformation in both loading directions to ensure a consistent starting position.

\subsection{Physiological rate tests}

As recounted in the introduction section, there does not appear to be a general consensus on the exact rate of deformation incurred by the valves in vivo (Doehring et al. 2004; LeesonDietrich et al. 1995; Sacks and Yoganathan 2007; Thubrikar et al. 1993). In order to avoid apprehension in the investigation of rate effects at physiological rates, we chose all three reported extension rates in the literature, which for a $12 \times 12 \mathrm{~mm}$ sample correspond to the following: (i) extension rate of $30 \mathrm{~mm} / \mathrm{s}$ for $\dot{\lambda}=2.5 \mathrm{~s}^{-1}$ as per Leeson-Dietrich et al. (1995); (ii) extension rate of $75 \mathrm{~mm} / \mathrm{s}$ for $\dot{\lambda}=6.25 \mathrm{~s}^{-1}$ as per Thubrikar et al. (1993) and Doehring et al. (2004); and (iii) extension rate of $148.8 \mathrm{~mm} / \mathrm{s}$ for $\dot{\lambda}=12.4 \mathrm{~s}^{-1}$ as per Sacks and Yoganathan (2007). We refer to all these three rates as the physiological rates in this paper.

Accordingly, equi-biaxial deformation rate tests were carried out on both AV and PV samples at $\dot{\lambda}=2.5,6.25$ and $12.4 \mathrm{~s}^{-1}$, up to failure, with six repeats for each test. However, in order to mimic the physiological deformation conditions, where the AV is known to endure strains of approximately $10.1 \%$ and $30.8 \%$ in the circumferential and radial directions in vivo, respectively, we also implemented a 1:3 biaxial deformation rate ratio protocol for each valvular specimen. Therefore, the corresponding stretch rates devised for each 1:3 rate ratio test are as follows: (i) extension rates of $10 \mathrm{~mm} / \mathrm{s}$ and $30 \mathrm{~mm} / \mathrm{s}$ resulting in stretch rates of $\dot{\lambda}=$ 0.83 and $2.5 \mathrm{~s}^{-1}$ in the circumferential and radial directions, respectively; (ii) extension rates of $25 \mathrm{~mm} / \mathrm{s}$ and $75 \mathrm{~mm} / \mathrm{s}$ resulting in stretch rates of $\dot{\lambda}=2.08$ and $6.25 \mathrm{~s}^{-1}$ in the circumferential and radial directions, respectively; (iii) extension rates of $49.6 \mathrm{~mm} / \mathrm{s}$ and $148.8 \mathrm{~mm} / \mathrm{s}$ resulting in stretch rates of $\dot{\lambda}=4.13$ and $12.4 \mathrm{~s}^{-1}$ in the circumferential and radial directions, respectively. 
Similar to the equi-biaxial rate tests, the 1:3 rate tests were repeated with six specimens per test.

\subsection{Sub physiological rate tests}

In order to investigate the effects of the rate of deformation on the mechanical properties of the semilunar valve, and to provide a comparative platform with the (Cauchy) stress-stretch curves obtained at high physiological rates, two sub-physiological deformation rates were also chosen to carry out the deformation tests at both equi-biaxial and 1:3 ratio deformation rates up to failure. These include: (i) extension rate of $12 \mathrm{~mm} / \mathrm{s}$ corresponding to $\dot{\lambda}=1 \mathrm{~s}^{-1}$ and (ii) extension rate of $0.012 \mathrm{~mm} / \mathrm{s}$ corresponding to $\dot{\lambda}=0.001 \mathrm{~s}^{-1}$ for equi-biaxial deformation rate tests; (iii) extension rates of $4 \mathrm{~mm} / \mathrm{s}$ and $12 \mathrm{~mm} / \mathrm{s}$ resulting in stretch rates of $\dot{\lambda}=0.33$ and 1 $\mathrm{s}^{-1}$ in the circumferential and radial directions, respectively; and (iv) extension rates of 0.004 $\mathrm{mm} / \mathrm{s}$ and $0.012 \mathrm{~mm} / \mathrm{s}$ resulting in stretch rates of $\dot{\lambda}=0.00033$ and $0.001 \mathrm{~s}^{-1}$ in the circumferential and radial directions, respectively, for 1:3 ratio biaxial deformation rate tests. All tests were repeated with six specimens.

\subsection{Statistical analysis}

Where reported, statistical analysis has been performed on the gradient of the obtained (Cauchy) stress-stretch $(\sigma-\lambda)$ curves; i.e. $\Delta \sigma / \Delta \lambda$, calculated numerically at $\sigma=50,100$ and $200 \mathrm{kPa}$. The value of $\Delta \sigma / \Delta \lambda$ was calculated numerically from the six repeat $\sigma-\lambda$ curves obtained in each test, at the designated (Cauchy) stress levels above. A Kolmogorov-Smirnov test using GraphPad Prism ${ }^{\circledR}$ was performed on each group first to check for the normality of the distribution of the gradient values. For groups which did or did not return a normal distribution, a one-tailed unpaired t-test or a Mann-Whitney test was performed, respectively, with statistical significance set at $p<0.05$. The sign ' $*$ ' in the plots/graphs is used to indicate the statistical significance between the compared datasets.

\section{Results}

As the first step, we start by reporting the strain rates experienced by the samples versus the applied rates. Note that the applied rates are the nominal rates set as a control variable input, 
while the experienced rates by the samples are calculated directly from the displacement of the markers at each recorded time increment a posteriori. Table 1 summarises these values, presented as average $\pm \mathrm{SD}$, calculated from the performed six repeat tests for each rate. Qualitatively, the calculated stretch rates are generally lower than the nominally applied rates. However, the trend of change in calculated rates is directly correlated with that of the applied, i.e. as the applied rates increase so do the calculated rates and vice versa.

\subsection{Cauchy stress - stretch $(\sigma-\lambda)$ curves for the samples tested at physiological rates}

In order to enable a distinction between the obtained $\sigma-\lambda$ curves under physiological versus sub-physiological rates, and allow a clearer comparison between the $\sigma-\lambda$ curves at the designated physiological rates in Section 2.1, we present the physiological rates' $\sigma-\lambda$ curves separately to the ones obtained from the sub-physiological rates.

Filtered $\sigma-\lambda$ curves for six AV and PV samples tested at the designated physiological rates are presented in Figures 2 and 3, respectively. The presented experimental data are filtered datasets, using a Savitzky-Golay filter, of the experimentally obtained $\sigma-\lambda$ curves.

From these, averaged data were calculated by averaging the stretch and stress values at each time step over the six repeats. The $\sigma-\lambda$ curves constructed from this averaging are shown in Figure 4 for both AV and PV specimens. The bars represent the standard deviation at the designated points on the curves. Analysis surrounding the trends and rate-effects will be conferred in Section 4. Note that the asterisk sign * indicates statistically significant difference between the gradients of the designated curves. Details are presented in Section 3.3.

\subsection{Cauchy stress - stretch $(\sigma-\lambda)$ curves for the samples tested at sub physiological rates}

Consistent with the presentation of results in the previous section, the filtered $\sigma-\lambda$ curves obtained from AV and PV specimens tested at the sub-physiological rate domains designated in Section 2.2 are shown in Figures 5 and 6, respectively. In the same way as the previous section, averaged $\sigma-\lambda$ curves were constructed from the averaged data over each six presented repeats. The ensuing averaged curves are shown in the bottom panels of Figures 5 
and 6 for the respective AV and PV samples. The bars represent the standard deviation at the designated points on the curves, and the asterisk sign $*$ indicates statistically significant difference between the gradients of the designated curves. Details are presented in Section 3.3.

\subsection{The gradients}

The statistical analysis pertaining to the change in the gradient of the $\sigma-\lambda$ curves with rate for $\dot{\lambda}=1 \mathrm{~s}^{-1}$ versus $\dot{\lambda}=0.001 \mathrm{~s}^{-1}$ rate of deformation tests was presented in our previous study (Anssari-Benam et al. 2019). Those results indicated statistically significant differences in curves' gradients across the two rates, showing a typical stiffening effect with increase in rate for $\sigma>50 \mathrm{kPa}$, in both $\mathrm{AV}$ and $\mathrm{PV}$ specimens. Here, therefore, we present the results for gradients of the new curves obtained under the tested physiological rates. The average $( \pm$ SD) gradient values established from each six repeats are shown in Figures 7 and 8 for PV and AV samples, respectively, calculated at $\sigma=50,100$ and $200 \mathrm{kPa}$. Note that the stress $\sigma$ endured by the AV at physiological function in vivo is thought to be between 160 to $200 \mathrm{kPa}$ (Hamid et al. 1985; Christie 1992), with studies also considering $240 \mathrm{kPa}$ (Stella et al. 2007) and up to around $300 \mathrm{kPa}$ (Rousseau et al. 1988). Noting that not all curves report stress levels of beyond 200 $\mathrm{kPa}$, here we only consider analysis up to $200 \mathrm{kPa}$, since stress levels beyond this mark appear to have little physiological relevance.

As the results indicate, in PV samples there is a statistically significant difference in the gradients of the $\sigma-\lambda$ curves obtained at $\dot{\lambda}=12.4 \mathrm{~s}^{-1}$ versus $\dot{\lambda}=6.25,2.5$ and $1 \mathrm{~s}^{-1}$ under equibiaxial as well as the ensuing 1:3 rate ratios (see Figure 7), at all stress levels except at $\sigma=50$ $\mathrm{kPa}$ under equi-biaxial rate loading. By extension, the statistical significance also exists between the gradients of the $\sigma-\lambda$ curves obtained at $\dot{\lambda}=12.4 \mathrm{~s}^{-1}$ versus those of $\dot{\lambda}=1 \mathrm{~s}^{-1}$ (and the ensuing 1:3 rate ratio). In AV samples, the gradients of the $\sigma-\lambda$ curves obtained at $\dot{\lambda}=12.4 \mathrm{~s}^{-1}$ become statistically significantly different from those obtained under $\dot{\lambda}=2.5 \mathrm{~s}^{-1}$ and $1 \mathrm{~s}^{-1}$, and the ensuing 1:3 rate ratios, for $\sigma \geq 100 \mathrm{kPa}$ (see Figure 8). No statistically significant difference was observed between the gradients of curves obtained at $\dot{\lambda}=6.25,2.5$ and $1 \mathrm{~s}^{-1}$ across any stress levels for either AV or PV samples. 


\section{Discussion}

The motivation by which the current work was predicated upon came from our previous work which, comparing the mechanical behaviour of the semilunar valves studied across sufficiently large deformation rates under sub-physiological domains, demonstrated a clear rate-dependent trend (Anssari-Benam et al. 2019). The natural next step was therefore to extend that work to the domain of physiological rates, to establish and document the potential rate-effects on the mechanical behaviour of AV and PV specimens under physiological loading conditions. The extensive set of data presented in this work reinforce our previous findings, and perhaps provide first insights into the rate-dependent mechanical behaviour of the valves under in vivo physiological loading rates. To our knowledge, in the arena of the biomechanics of semilunar valves, this premise has been put forward for the first time here.

\subsection{Comparison of the $\sigma-\lambda$ curves at physiological rates}

As recounted earlier in this paper, the literature suggests several values for the physiological stretch rate endured by the $\mathrm{AV}$ in vivo, ranging from $\dot{\lambda}=2.5$ to $6.25 \mathrm{~s}^{-1}$ and as high as $\dot{\lambda}=12.4 \mathrm{~s}^{-1}$. Therefore, we undertook to investigate the mechanical behaviour of the valves under biaxial deformation across all these rates. When comparing the obtained $\sigma-\lambda$ curves, it is clear that there are no significant differences between the observed behaviours under $\dot{\lambda}=2.5$ and $6.25 \mathrm{~s}^{-1}$. This can be seen from the graphs presented in Figures 2 to 4 . The statistical analysis on the gradient of the curves also reinforces this observation, as no significant difference was established between the gradients of the $\sigma-\lambda$ curves obtained at those rates. This observation perhaps may not be surprising, as stretch rates 2.5 and $6.25 \mathrm{~s}^{-1}$ are reasonably close to one another, particularly when viewed from the perspective of the actual rates endured by the samples, as presented in Table 1 . The gap between the two rates is sufficiently small so that it does not merit a significant difference in the mechanical behaviour of the tissue samples. Note the overlap between the $\sigma-\lambda$ curves at 2.5 and $6.25 \mathrm{~s}^{-1}$, likely due to the natural sampleto-sample variability or load readings uncertainty at the initial range of deformation. However, when compared with the $\sigma-\lambda$ curves obtained at $\dot{\lambda}=12.4 \mathrm{~s}^{-1}$, which is in a higher order of magnitude compared with the two previous rates, the rate effects become noticeable. This noticeable difference may be observed more readily from the averaged data curves in Figure 4, 
as well as the statistically significant difference in the gradient of the $\sigma-\lambda$ curves at $\dot{\lambda}=12.4$ $\mathrm{s}^{-1}$ versus those of the $\dot{\lambda}=2.5$ and $6.25 \mathrm{~s}^{-1}$ curves, as shown in Figures 7 and 8 .

\subsection{Comparison of the $\sigma-\lambda$ curves at physiological versus sub physiological rates}

For the benefit of direct comparison, the averaged-data $\sigma-\lambda$ curves obtained at all rates for both AV and PV specimens are shown in Figure 9. It is readily observed that qualitatively, the $\sigma-\lambda$ curves show a clear rate-dependency, from the quasi-static base-line to the maximum physiological rate reported. We refer to the curves obtained at $\dot{\lambda}=0.001 \mathrm{~s}^{-1}$ as the base-line curve, where below this rate $\sigma-\lambda$ curves become intractable to rate-effects. For materials that exhibit stress-relaxation, such as heart valves (see, e.g., Stella et al. 2007), a characteristic relaxation time $\tau$ may be defined whereby $99 \%$ of the relaxation fades within $5 \tau$. Therefore, if the deformation rate is chosen sufficiently low to allow enough time for the viscous processes to take effect and fade, the ensuing $\sigma-\lambda$ curves may be deemed intractable to further reductive viscous effects. Such a curve may therefore provide a baseline for comparing the rate-effects with (Pioletti and Rakotomanana 2000). Based on our previous stress-relaxation tests on AV samples (Anssari-Benam et al. 2011b), and the calculations presented in (Anssari-Benam et al. 2017), $\dot{\lambda}=0.001 \mathrm{~s}^{-1}$ provides a sufficiently slow deformation to be considered as the base-line response. Therefore, while tests at $\dot{\lambda}=0.001 \mathrm{~s}^{-1}$ may not be physiologically relevant, they provide useful information from the perspective of material characterisation and rate-effects analysis.

In our preceding publication, we presented a detailed analysis on the existence of statistically significant rate-effects between the $\sigma-\lambda$ curves obtained at $\dot{\lambda}=1 \mathrm{~s}^{-1}$ versus that of $\dot{\lambda}=0.001$ $\mathrm{s}^{-1}$ (Anssari-Benam et al. 2019). We therefore refrain from replicating such analysis here. Based on the results of that study, and the reported averaged curves, a clear rate-dependent trend between the $\sigma-\lambda$ curves obtained at $\dot{\lambda}=2.5$ and $6.25 \mathrm{~s}^{-1}$ (and the corresponding 1:3 rate ratio) versus those of $\dot{\lambda}=0.001 \mathrm{~s}^{-1}$ is evident. By extension, the $\sigma-\lambda$ curves obtained at $\dot{\lambda}=$

$12.4 \mathrm{~s}^{-1}$ versus those of $\dot{\lambda}=0.001 \mathrm{~s}^{-1}$ (and the corresponding 1:3 rate ratio) also show a significant rate-effect.

We note that when comparing the $\sigma-\lambda$ curves obtained at $\dot{\lambda}=1$ and $0.001 \mathrm{~s}^{-1}$ in this study with those of our previous study in (Anssari-Benam et al. 2019), slight differences particularly 
in the initial (soft) region of the curves may be noticed. This is likely due to the adjustments that needed to be made to the grips to facilitate the higher rate tests in this study, as well as the image recording equipment, compared with the setup used in our previous study. In addition, the natural sample-to-sample variability may have also played its part. Therefore, in order to provide consistency, we carried out the equi-biaxial rate tests at $\dot{\lambda}=1$ and $0.001 \mathrm{~s}^{-1}$ again in this study too. Nevertheless, both our previous and current studies demonstrate the same rateeffect: with increase in the deformation rate, semilunar valves exhibit stiffening as reflected in their $\sigma-\lambda$ curves (see also Figure 9).

\subsection{Implications for future developments}

The results of this study provide a comprehensive set of data for material and rate-dependent behaviour characterisation of the semilunar valves, from quasi-static to physiological rates across a 10,000 fold range, demonstrating rate-dependency in the mechanical behaviour of the valves. These results may therefore motivate investigation of such behaviour in other heart valves, namely the atrioventricular valves. Recent studies have provided first steps in investigating the rate-effects in the mechanical behaviour of those valves (see, e.g., Jett et al. 2018); however, the employed range of rates (i.e. 4.42 to $7.92 \mathrm{~N} / \mathrm{min}$ ) may not commission a sufficiently large span to meaningfully document rate effects. Additionally, the physiological rates appear to be above the rates employed for the mechanical behaviour of heart valves (e.g., 2.29 N/min in Laurence et al. 2019). In the arena of semilunar heart valves, in specific, our results may also provide a comprehensive benchmark for comparing the mechanical behaviour of tissue-engineered versus native valves. A recent study on modelling the behaviour of tissueengineered AVs employs a rate of $0.02 \mathrm{~s}^{-1}$ (Noble et al. 2019), citing preceding studies using the same range of rates. In view of the current study, the documented mechanical behaviour at $0.02 \mathrm{~s}^{-1}$ may not be directly representative of the in vivo behaviour. Therefore, the outcomes of this study may have far reaching effects in devising mechanical testing protocols in order to appropriately capture and characterise the rate-dependent mechanical behaviour of heart valves.

The same notion may be extended to developing continuum and computational models that portend the rate-dependent mechanical behaviour of semilunar valves. Most developed models for application to heart valves to date have been established on the principles of hyperelasticity, 
or more broadly on discounting the rate-effects. Those models may accurately describe the behaviour of the specimens at, or within a close proximity of, the rates under which the mechanical tests were carried out. However, it is clear that such models fail to appropriately characterise the true rate-dependent behaviour of the valves. We have previously presented a modelling framework within which the rate-dependent behaviour of the AV tissue may be accounted for by incorporating the rate of deformation as an explicit modelling parameter (Anssari-Benam et al. 2017; 2018). The results of the current study further reinforce the requisite for countenancing such models, or developing alternative models to address the ratedependent behaviour. Such models, at the very least, will allow approximation or prediction of the mechanical behaviour of the specimens at higher rates from the experimental data obtained at lower rates. This may be viewed as a particularly useful feature when due to experimental and technical limitations, performing tests at higher physiological rates is not possible.

\section{Conclusion}

The mechanical behaviour of semilunar heart valves is rate-dependent, from quasi-static to the physiological range of loading rate, demonstrably manifested when the specimens are tested across sufficiently large deformation rates. From the results of this study, the 'sufficiently large' span may be considered as at least a difference in the order of magnitude, since the $\sigma-\lambda$ curves obtained at $\dot{\lambda}=6.25,2.5$ and $1 \mathrm{~s}^{-1}$ (including their ensuing 1:3 deformation rate ratios) did not show a statistically significant difference. However, compared with the $\sigma-\lambda$ curves obtained at $\dot{\lambda}=12.4 \mathrm{~s}^{-1}$, statistically significant differences were documented versus the $\sigma-\lambda$ curves obtained at $\dot{\lambda}=2.5$ and $1 \mathrm{~s}^{-1}$, and by extension with $\sigma-\lambda$ curves obtained at $\dot{\lambda}=0.001 \mathrm{~s}^{-1}$. Therefore, even across deformation rates that are considered as physiological, i.e. $2.5 \mathrm{~s}^{-1} \leq \dot{\lambda} \leq 12.4 \mathrm{~s}^{-1}$, semilunar valve specimens exhibit a rate-dependent behaviour if the deformation rate span is large enough.

While further investigation may be required, a preliminary observation of our results may conclude that the $\sigma-\lambda$ curves of the semilunar valve specimens may perhaps tend to cluster at $\dot{\lambda} \geq 1 \mathrm{~s}^{-1}$, to the effect that there are no statistically significant differences between $\sigma-\lambda$ curves obtained at $\dot{\lambda}=1,2.5$ or $6.25 \mathrm{~s}^{-1}$. Within this clustering, however, curves obtained at $\dot{\lambda}=12.4 \mathrm{~s}^{-1}$ still possess a statistically significant difference to those obtained at $\dot{\lambda}=6.25,2.5$ or $1 \mathrm{~s}^{-1}$. Deformation tests performed at lower rates may therefore risk significantly 
underestimating the true mechanical behaviour of semilunar valves. Based on our results, in order to get closer to the true mechanical behaviour of these heart valves, tests may not be performed at $\dot{\lambda}<1 \mathrm{~s}^{-1}$.

The results presented here may serve as a comprehensive set of data for constitutive modelling of the semilunar valves, from quasi-static to physiological deformation rates. The results also underline the need for developing appropriate models for capturing this rate-dependent behaviour, currently being overlooked in the literature. The premise put forward in this study may be used to investigate and appropriately characterise the mechanical behaviour of other heart valves.

\section{Data accessibility}

The $\sigma-\lambda$ data presented in this study are available at the University of Portsmouth research data repository: https://doi.org/10.17029/855847b3-c9d3-4a1a-be9d-c71cc4a97eb5

\section{Competing interests}

The authors have no conflict of interest to declare.

\section{Acknowledgements}

This work was part funded by a Leverhulme Trust grant (VP1-2017-001). 


\section{References}

Anssari-Benam, A., Bader, D.L., Screen, H.R.C., 2011a. A combined experimental and modelling approach to aortic valve viscoelasticity in tensile deformation. J. Mater. Sci.: Mater. Med. 22, 253-262.

Anssari-Benam, A., Bader, D.L., Screen, H.R.C., 2011b. Anisotropic time-dependant behaviour of the aortic valve. J. Mech. Behav. Biomed. Mater. 4, 1603-1610.

Anssari-Benam, A., Bucchi, A., Screen, H.R.C., Evans, S.L., 2017. A transverse isotropic viscoelastic constitutive model for aortic valve tissue. R. Soc. Open Sci. 4, 160585. (doi: $10.1098 /$ rsos.160585)

Anssari-Benam, A., Tseng, Y.-T., Bucchi, A., 2018. A transverse isotropic constitutive model for the aortic valve tissue incorporating rate-dependency and fibre dispersion: application to biaxial deformation. J. Mech. Behav. Biomed. Mater. 85, 80-93.

Anssari-Benam, A., Tseng, Y.-T., Holzapfel, G.A., Bucchi, A., 2019. Rate-dependency of the mechanical behaviour of semilunar heart valves under biaxial deformation. Acta Biomater. 88, 120-130.

Christie, G.W., 1992. Anatomy of aortic heart valve leaflets: the influence of glutaraldehyde fixation on function. Eur. J. Cardio-thorac. Surg. 6, S25-S33.

Doehring, T.C., Carew, E.O., Vesely, I., 2004. The effect of strain rate on the viscoelastic response of aortic valve tissue: a direct-fit approach. Ann. Biomed. Eng. 32, 223-232.

Grashow, J.S., Yoganathan, A.P., Sacks, M.S., 2006. Biaxial stress-stretch behavior of the mitral valve anterior leaflet at physiologic strain rates. Ann. Biomed. Eng. 34, 315-325.

Hamid, M.S., Sabbah, H.N., Stein, P.D., 1985. Finite element evaluation of stresses on closed leaflets of bioprosthetic heart valves with flexible stents. Finite Elem. Anal. Des. 1, 213-225. 
Jett, S., Laurence, D., Kunkel, R., Babu, A.R., Kramer, K., Baumwart, R., Towner, R., Wu, Y., Lee, C.H., 2018. An investigation of the anisotropic mechanical properties and anatomical structure of porcine atrioventricular heart valves. J. Mech. Behav. Biomed. Mater. 87, 155-171.

Laurence, D., Ross, C., Jett, S., Johns, C., Echols, A., Baumwart, R., Towner, R., Liao, J., Bajona, P., Wu, Y., Lee, C.H., 2019. An investigation of regional variations in the biaxial mechanical properties and stress relaxation behaviors of porcine atrioventricular heart valve leaflets. J. Biomech. 83, 16-27.

Leeson-Dietrich, J., Boughner, D., Vesely, I., 1995. Porcine pulmonary and aortic valves: a comparison of their tensile viscoelastic properties at physiological strain rates. J. Heart Valve Dis. 4, 88-94.

Noble, C., Choe, J., Uthamaraj, S., Deherrera, M., Lerman, A., Young, M., 2019. In silico performance of a recellularized tissue engineered transcatheter aortic valve. J. Biomech. Eng. $141,061004$.

Pioletti, D.P., Rakotomanana, L.R., 2000. Non-linear viscoelastic laws for soft biological tissues. Eur. J. Mech. A Solids 19, 749-759.

Rousseau, E.P.M., van Steenhoven, A.A., Janssen, J.D., 1988. A mechanical analysis of the closed Hancock heart valve prosthesis. J. Biomechanics 21, 545-562.

Sacks, M.S., Yoganathan, A.P., 2007. Heart valve function: a biomechanical perspective. Philos. Trans. R. Soc. Lond. B Biol. Sci. 362, 1369-1391.

Stella, J.A., Liao, J., Sacks, M.S., 2007. Time-dependent biaxial mechanical behavior of the aortic heart valve leaflet. J. Biomech. 40, 3169-3177.

Thubrikar, M.J., Heckman, J.L., Nolan, S.P., 1993. High speed cine-radiographic study of aortic valve leaflet motion. J. Heart Valve Dis. 2, 653-661. 
Table 1

\begin{tabular}{|c|c|c|c|c|c|c|c|c|}
\hline \multirow[b]{4}{*}{ Applied $\dot{\lambda}\left[\mathbf{s}^{-1}\right]$} & \multicolumn{8}{|c|}{$\dot{\lambda}\left[\mathrm{s}^{-1}\right]$ at the central region of the specimens } \\
\hline & \multicolumn{4}{|c|}{$\mathbf{A V}$} & \multicolumn{4}{|c|}{ PV } \\
\hline & \multicolumn{2}{|c|}{ Equi-biaxial } & \multicolumn{2}{|c|}{$1: 3$ rate } & \multicolumn{2}{|c|}{ Equi-biaxial } & \multicolumn{2}{|c|}{ 1:3 rate } \\
\hline & Cir. & Rad. & Cir. & Rad. & Cir. & Rad. & Cir. & Rad. \\
\hline $12.4(4.13: 12.4)$ & $9.30 \pm 1.03$ & $10.53 \pm 2.41$ & $2.53 \pm 0.52$ & $10.32 \pm 2.32$ & $9.42 \pm 1.61$ & $10.13 \pm 1.89$ & $2.79 \pm 0.42$ & $10.07 \pm 1.56$ \\
\hline $6.25(2.08: 6.25)$ & $4.25 \pm 1.40$ & $5.32 \pm 1.15$ & $1.61 \pm 0.33$ & $5.58 \pm 1.26$ & $4.33 \pm 0.86$ & $5.83 \pm 2.19$ & $1.98 \pm 0.59$ & $6.09 \pm 1.08$ \\
\hline $2.5(0.83: 2.5)$ & $1.79 \pm 0.42$ & $2.11 \pm 1.35$ & $0.75 \pm 0.28$ & $2.70 \pm 1.30$ & $1.56 \pm 1.06$ & $2.57 \pm 1.03$ & $0.70 \pm 0.12$ & $2.55 \pm 0.47$ \\
\hline $1(0.33: 1)$ & $0.57 \pm 0.05$ & $0.83 \pm 0.15$ & $0.36 \pm 0.10$ & $0.90 \pm 0.17$ & $0.69 \pm 0.09$ & $1.06 \pm 0.15$ & $0.40 \pm 0.06$ & $1.04 \pm 0.14$ \\
\hline $\begin{array}{l}0.001 \\
(0.00033: 0.001)\end{array}$ & $\begin{array}{c}0.0006 \\
\pm 0.0003\end{array}$ & $\begin{array}{c}0.0018 \\
\pm 0.0007\end{array}$ & $\begin{array}{c}0.0003 \\
\pm 0.0001\end{array}$ & $\begin{array}{c}0.0014 \\
\pm 0.0004\end{array}$ & $\begin{array}{c}0.0009 \\
\pm 0.0004\end{array}$ & $\begin{array}{c}0.0017 \\
\pm 0.0004\end{array}$ & $\begin{array}{c}0.0004 \\
\pm 0.0002\end{array}$ & $\begin{array}{c}0.0013 \\
\pm 0.0005\end{array}$ \\
\hline
\end{tabular}

Table 1 - Corresponding stretch rates in the central region of the specimens under equi-biaxial and $1: 3$ rate ratio in both loading directions. 


\section{Figure 1}
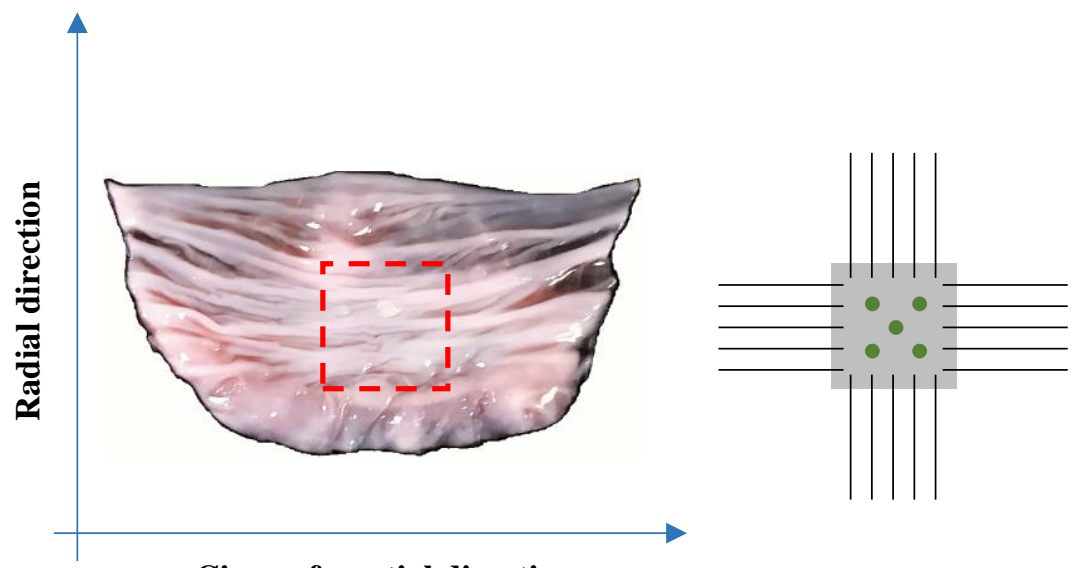

Circumferential direction

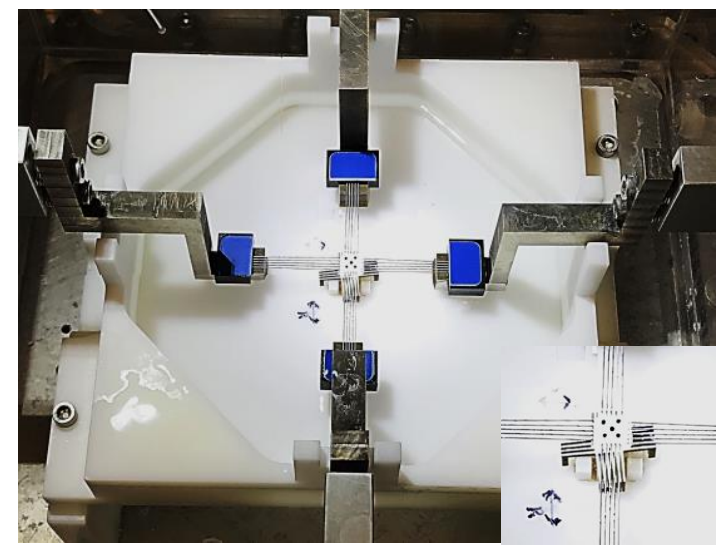

Figure 1 - The panels from left to right: a typical intact porcine AV leaflet from which a square specimen $(12 \times 12 \mathrm{~mm})$, shown by dashed lines, is prepared from its central region; a schematic of a square sample secured using tines and with five ink-markers printed on; a sample mounted on the ElectroForce ${ }^{\circledR}$ planar biaxial TestBench device using a custom-designed sample gripping mechanism consisting of BioRake (CellScale ${ }^{\circledR}$ ) tines. The inset in the right panel shows a zoomed view of the mounted sample. 
Figure 2
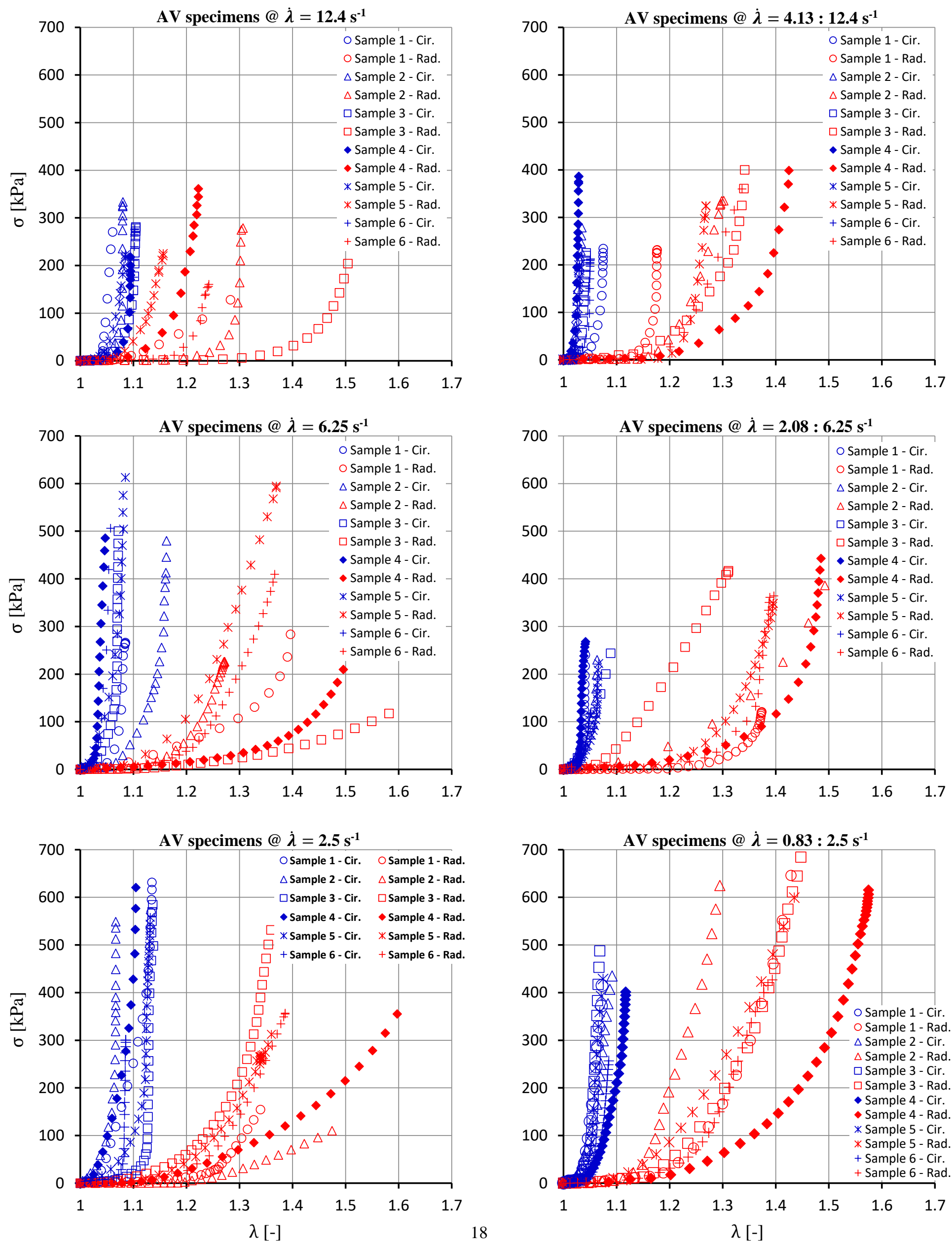
Figure 2 - Cauchy stress-stretch $(\sigma-\lambda)$ curves for AV samples (six repeats) tested at the three designated physiological rates. Plots in the left-hand side panels represent the equi-biaxial rate experiments, while the right-hand side planes show the results for 1:3 rate ratio tests. 
Figure 3
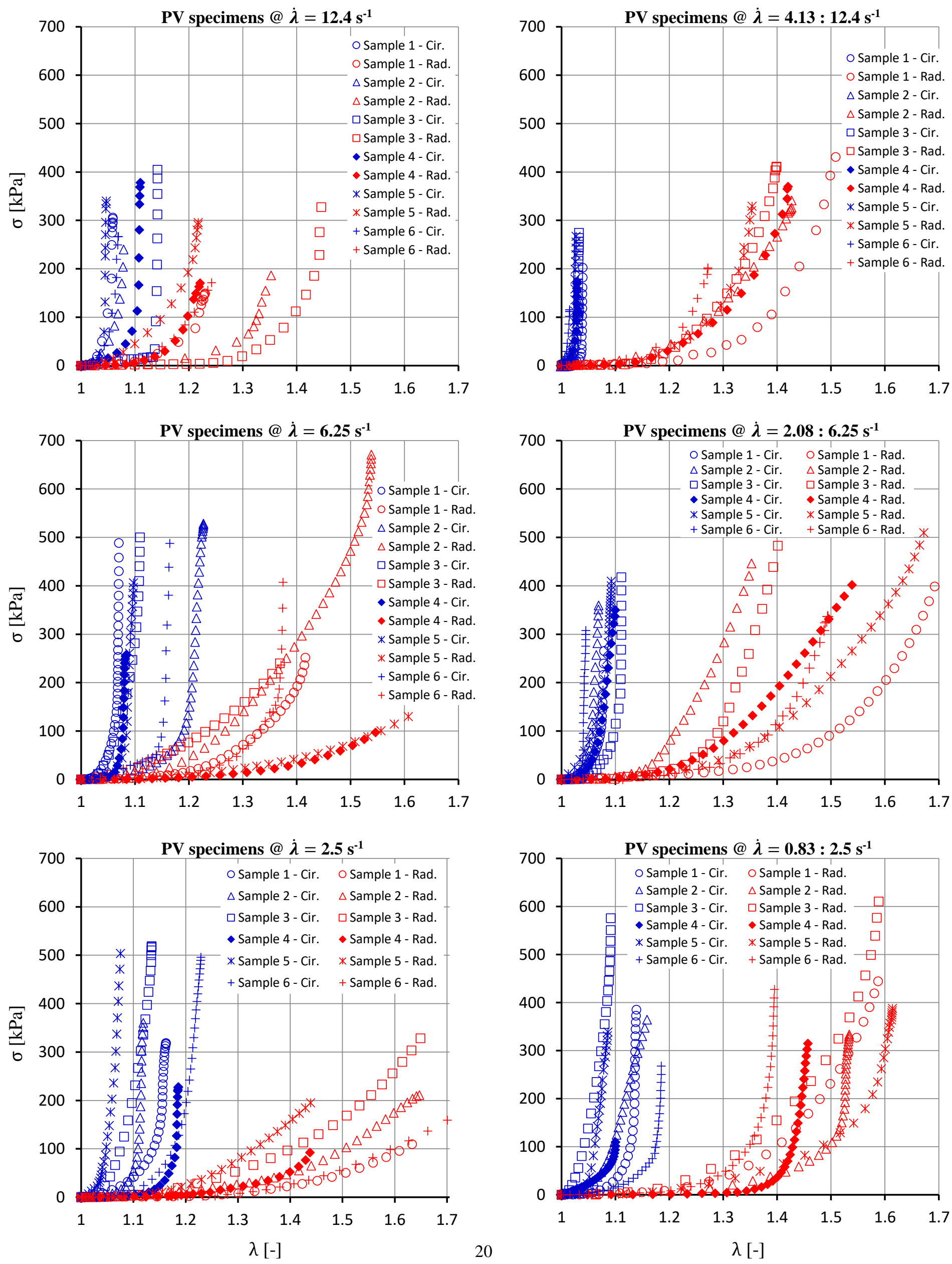
Figure 3 - Cauchy stress-stretch $(\sigma-\lambda)$ curves for PV samples (six repeats) tested at the three designated physiological rates. Plots in the left-hand side panels represent the equi-biaxial rate experiments, while the right-hand side planes show the results for 1:3 rate ratio tests. 


\section{Figure 4}
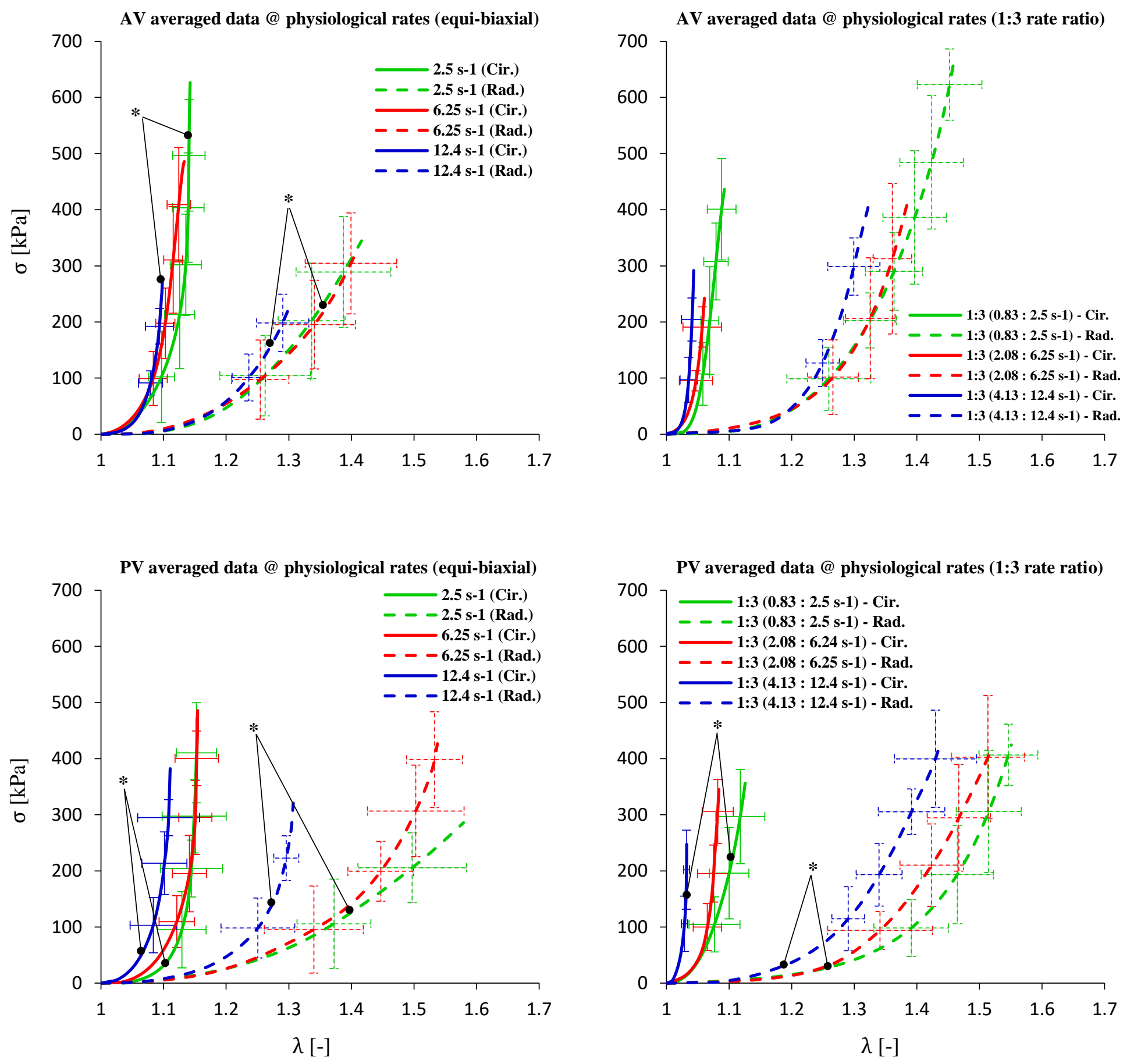
Figure 4 - Averaged data as representative $\sigma-\lambda$ curves for AV and PV specimens at each tested physiological rate under both equi-biaxial and 1:3 rate ratio. Top panels show the averaged data curves for AV specimens while the bottom panels present the results for PV samples. Straight and dashed curves represent the data in the circumferential and radial directions, respectively. The bars illustrate the standard deviation (SD) at each designated point. Pair of curves labelled by an asterisk sign * are statistically significantly different at stress levels $\sigma \geq 100 \mathrm{kPa}$ and $\sigma \geq 50 \mathrm{kPa}$ for $\mathrm{AV}$ and $\mathrm{PV}$ specimens, respectively. 
Figure 5
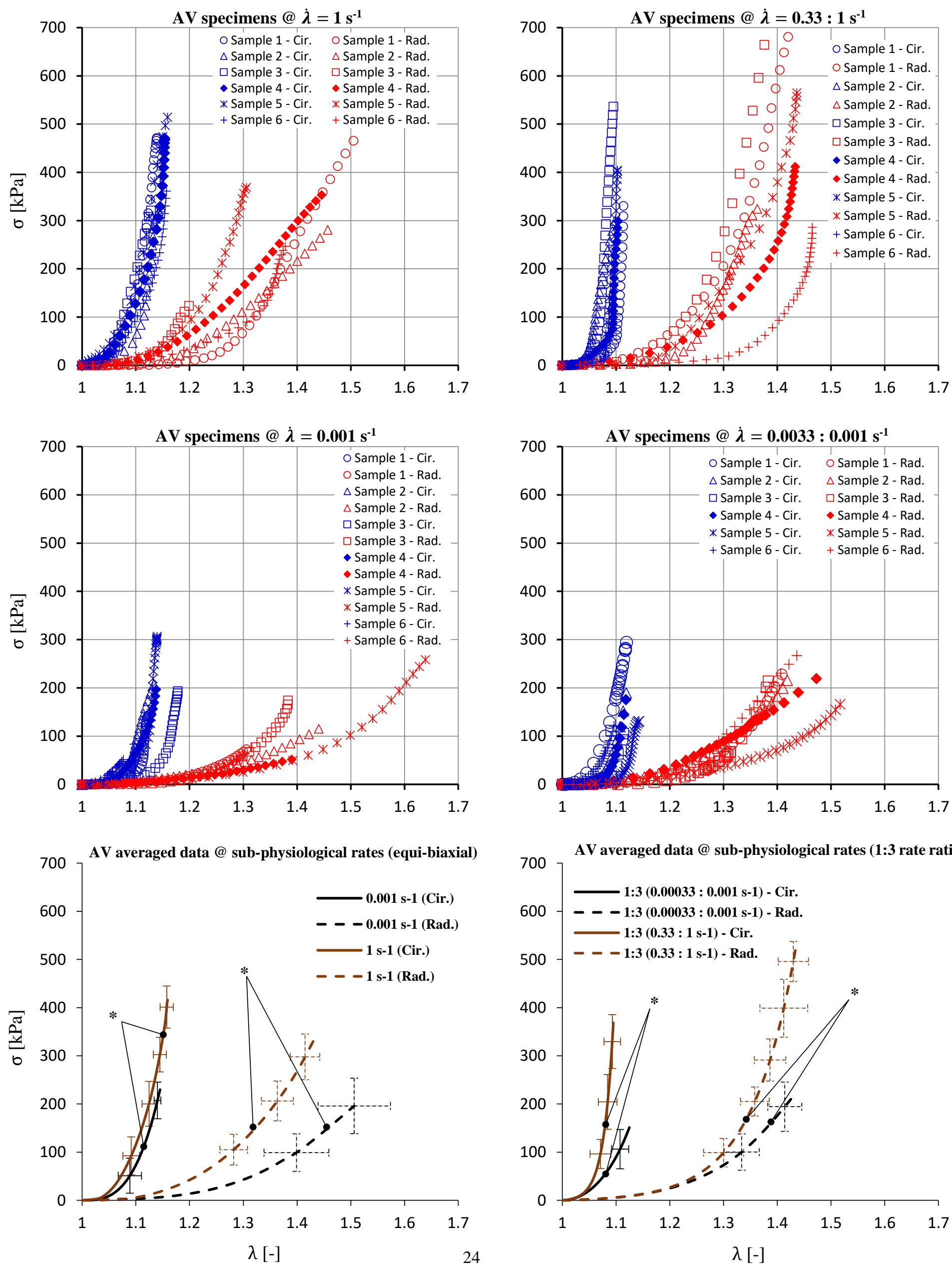
Figure 5 - Cauchy stress-stretch $(\sigma-\lambda)$ curves for AV samples (six repeats) tested at the two designated sub-physiological rates. Plots in the left-hand side panels represent the equi-biaxial rate experiments, while the right-hand side planes show the results for 1:3 rate ratio tests. The two bottom panels present the averaged data as representative $\sigma-\lambda$ curves at each tested rate under both equi-biaxial and 1:3 rate ratio. Straight and dashed curves represent the data in the circumferential and radial directions, respectively. The bars illustrate the standard deviation (SD) at each designated point. Pair of curves labelled by an asterisk sign * are statistically significantly different at stress levels $\sigma>50 \mathrm{kPa}$. 
Figure 6
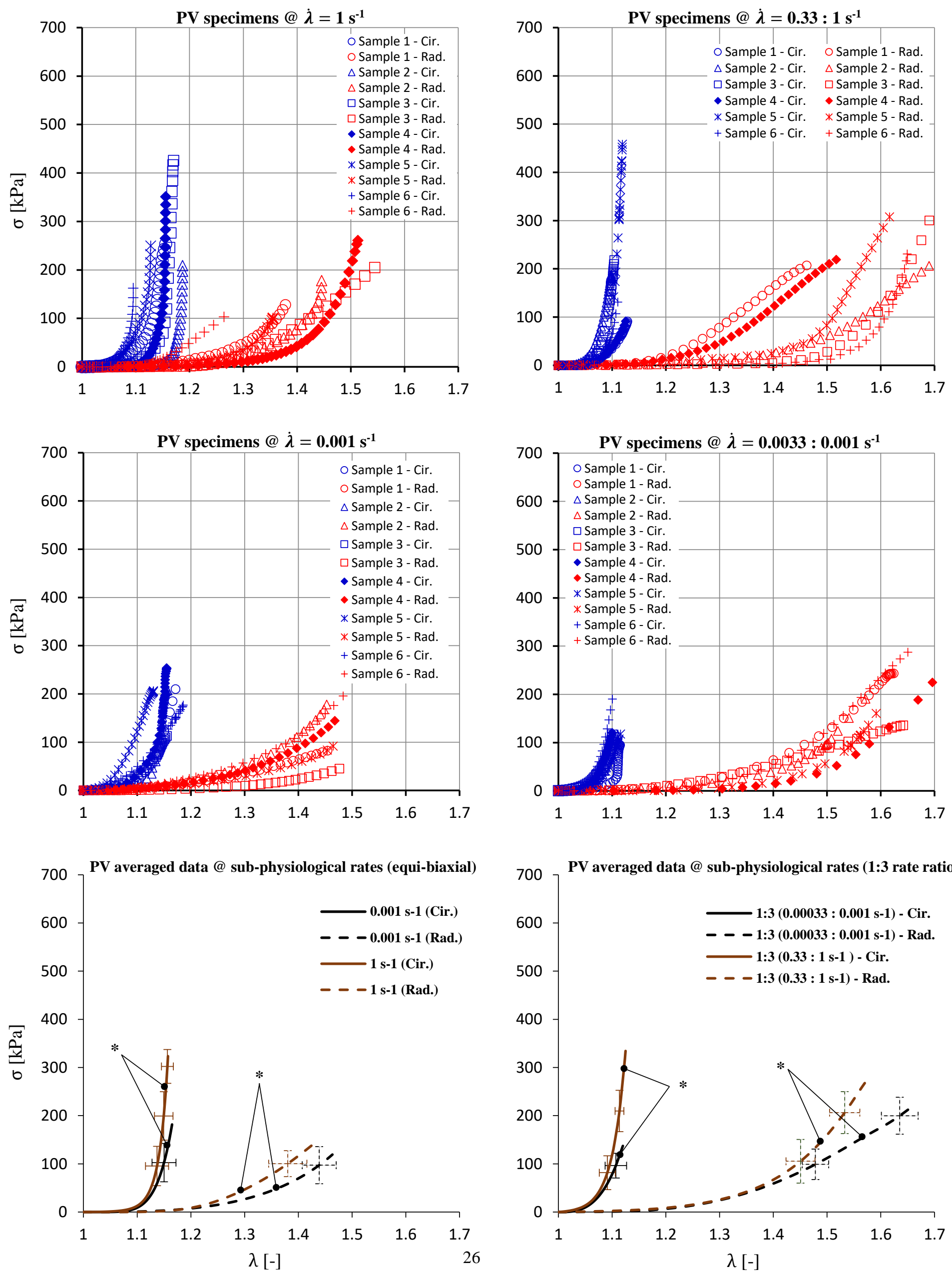
Figure 6 - Cauchy stress-stretch $(\sigma-\lambda)$ curves for PV samples (six repeats) tested at the two designated sub-physiological rates. Plots in the left-hand side panels represent the equi-biaxial rate experiments, while the right-hand side planes show the results for 1:3 rate ratio tests. The two bottom panels present the averaged data as representative $\sigma-\lambda$ curves at each tested rate under both equi-biaxial and 1:3 rate ratio. Straight and dashed curves represent the data in the circumferential and radial directions, respectively. The bars illustrate the standard deviation (SD) at each designated point. Pair of curves labelled by an asterisk sign * are statistically significantly different at stress levels $\sigma>50 \mathrm{kPa}$. 


\section{Figure 7}
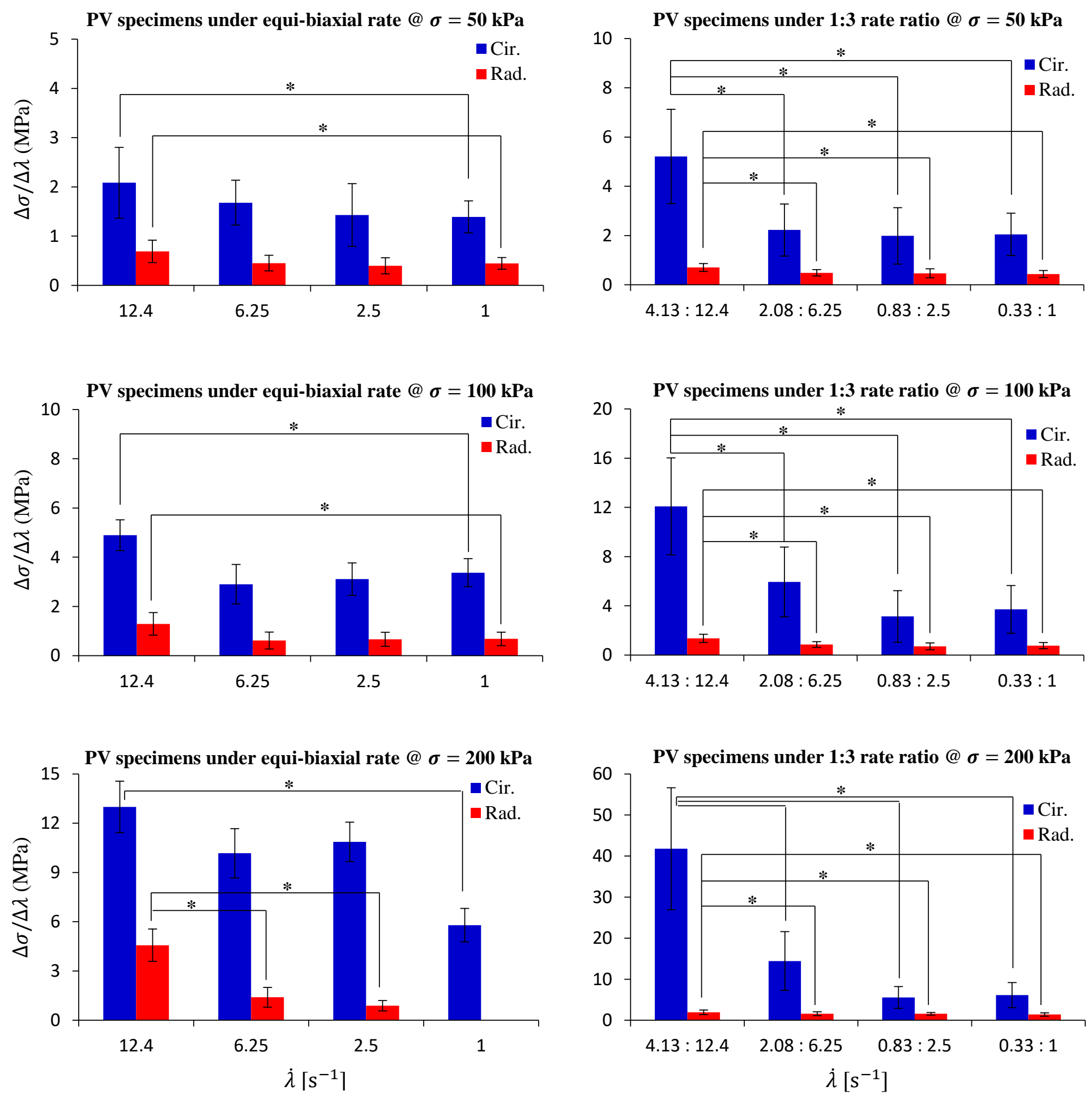

Figure 7 - Gradients $(\Delta \sigma / \Delta \lambda)$ calculated numerically from PV $\sigma-\lambda$ curves (six repeats) at each rate. Blue and red bars represent the values in circumferential and radial directions, respectively (for the coloured graphs see the on-line version). For presentation purposes, note that the $y$ axes are not in the same scale. The asterisk sign * represents statistical significance. 
Figure 8
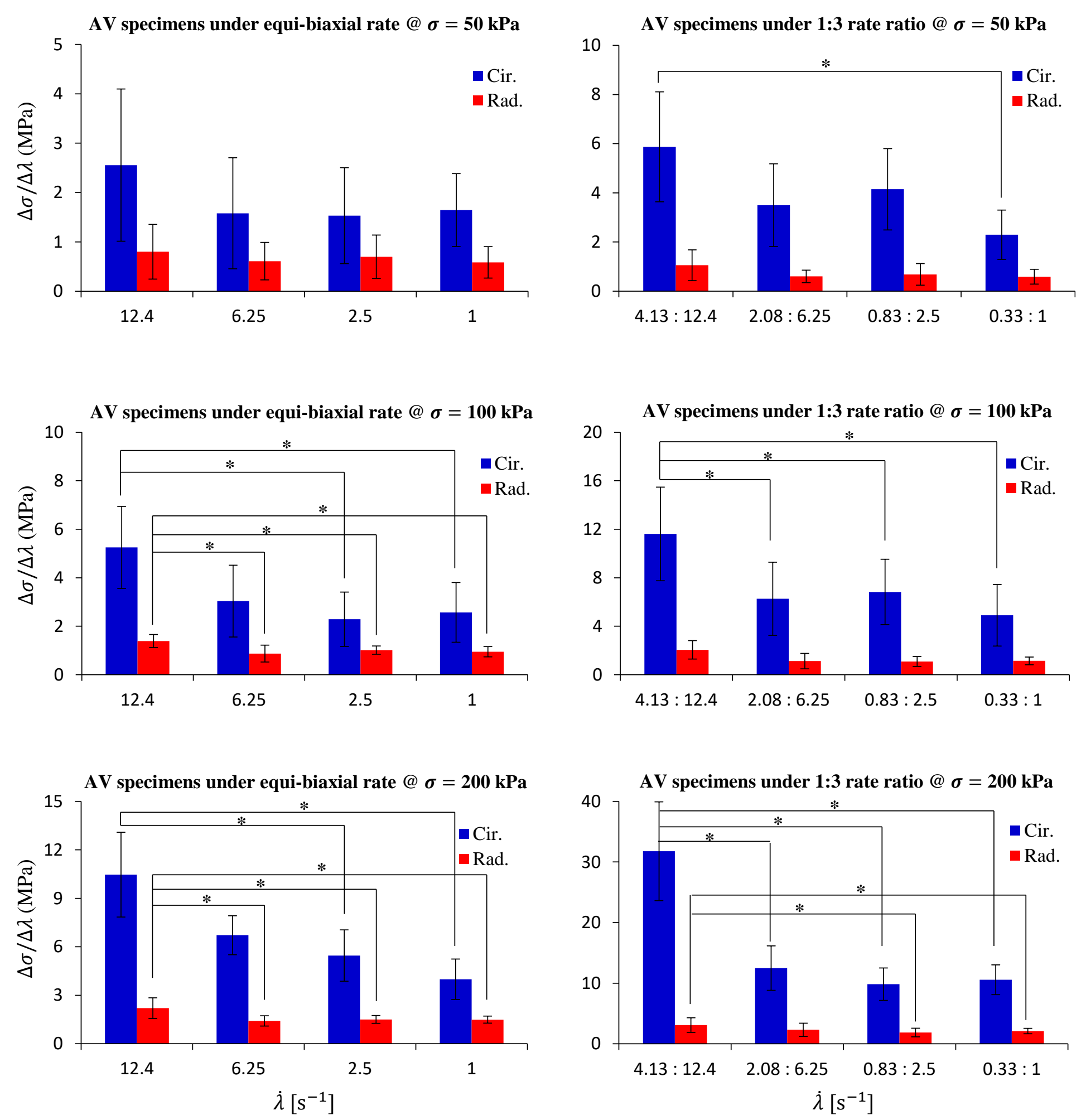

Figure 8 - Gradients $(\Delta \sigma / \Delta \lambda)$ calculated numerically from AV $\sigma-\lambda$ curves (six repeats) at each rate. Blue and red bars represent the values in circumferential and radial directions, respectively (for the coloured graphs see the on-line version). For presentation purposes, note that the $y$ axes are not in the same scale. The asterisk sign * represents statistical significance. 
Figure 9
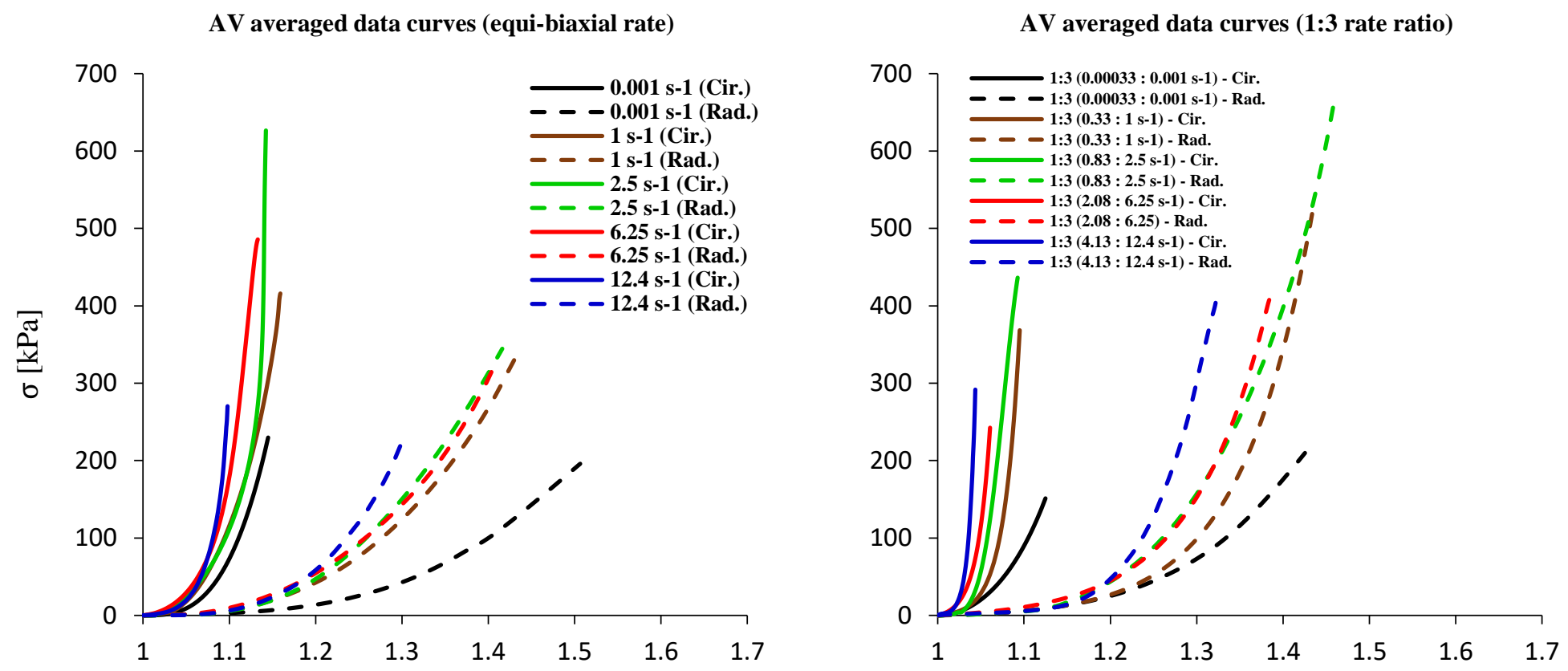

PV averaged data curves (equi-biaxial rate)
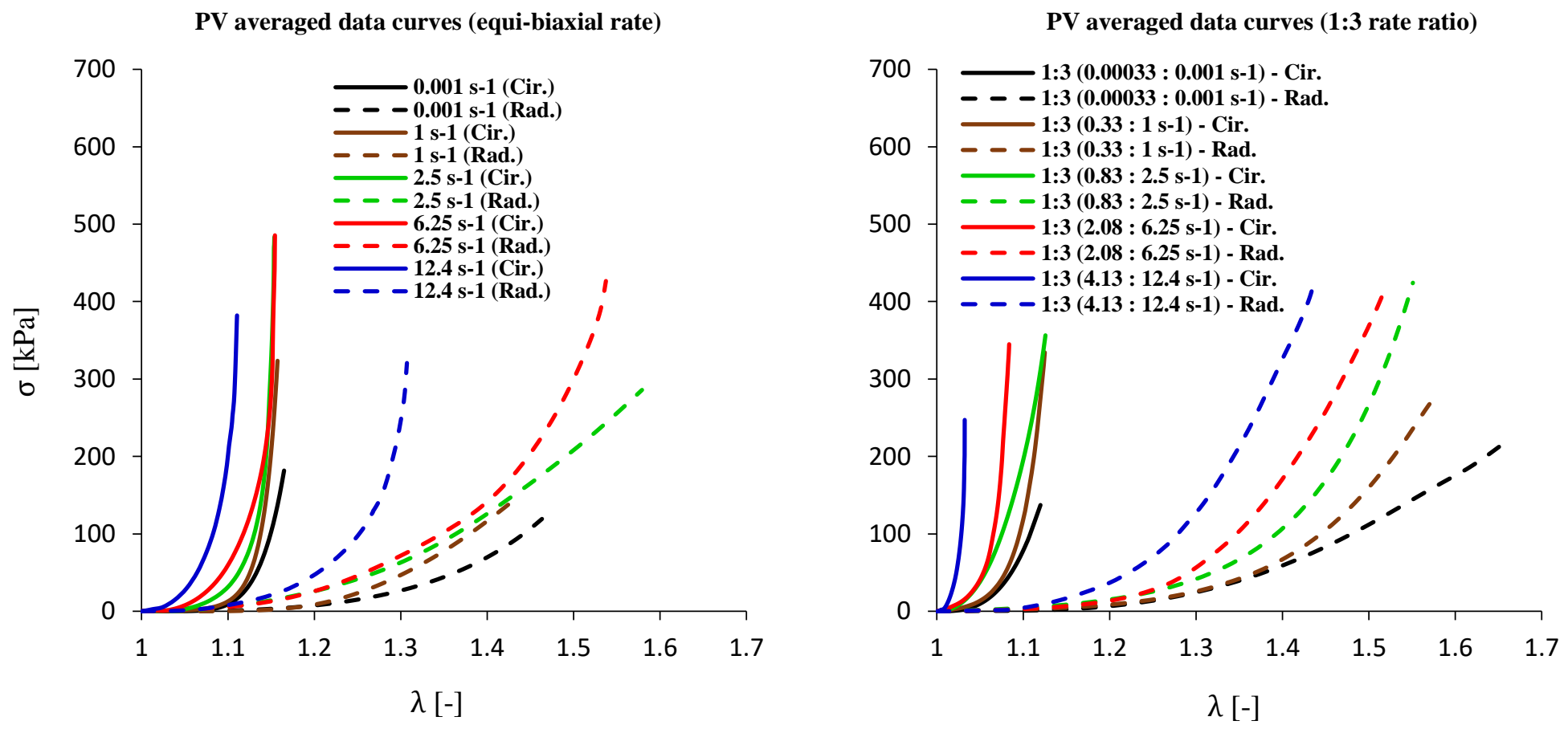

Figure 9-Averaged data curved for AV and PV specimens across all tested deformation rates.

The panels on the left-hand side represent the equi-biaxial rate results, while the right-hand side panel show the results for the corresponding 1:3 rate ratios. For clarity of presentation, the SD bars have been omitted. The continuous and dashed curves illustrate the circumferential and radial direction responses, respectively. For the coloured plots see the on-line version. 